\title{
Performances of the Main Beam Quadrupole Type1 Prototypes for CLIC
}

\author{
M. Modena, A. Bartalesi, J. Garcia Perez, R. Leuxe, G. Perrin-Bonnet, C. Petrone, M. Struik, and A. Vorozhtsov
}

\begin{abstract}
A critical magnet family for the future Compact Linear Collider (CLIC) is the Main Beam Quadrupole (MBQ) one. These magnets, placed along the two main linacs, will be actively stabilized in the nanometre range and are one of the key elements for reaching the outstanding nanometric dimensions and luminosity of the colliding beams. In the framework of the CLIC R\&D and prototypes procurement for the CLIC Test Facility under construction at CERN, several prototypes of $M B Q$ were procured. The MBQ magnet has a classical electro-magnetic design. A challenging aspect of the design is the extremely high mechanical precision required for the manufacturing and assembly of the iron quadrants. The challenging manufacturing aspects are presented and discussed. Results on the realized prototypes are discussed.
\end{abstract}

Index Terms-Accelerator magnets, magnetic measurements, precision engineering, resistive magnets.

\section{INTRODUCTION}

$\mathbf{T}$ HE Compact Linear Collider (CLIC) is an electronpositron linear collider project for a maximum energy of $3 \mathrm{TeV}$ and very high accelerating gradient of $100 \mathrm{MV} / \mathrm{m}$ [1]. In order to optimize the production of the needed radio frequency (RF) power for this high gradient, CLIC relies upon a two-beam acceleration concept: a $12 \mathrm{GHz}$ RF power is generated by a high current electron beam (drive beam) running parallel to the main beam. Along the drive beam linacs, the beam is decelerated in special Power Extraction and Transfer Structures (PETS), and the generated RF power is transferred to the accelerating structures of the main beam linacs. To provide the main beam focusing, a total of 4020 Main Beam Quadrupoles (MBQ) is required. In reference [2] are reported details of the design and $*$ of the different types of MBQ quadrupoles. The two-beams linac configuration consists of a repeated modular structure of five different types of so called "Modules". Along the main beam line, modules Type 0 have no MBQ but only accelerating Structures. In modules Type 1 to Type 4 the main difference is the length of the MBQ; where the MBQ are shorter, the space is filled with Acceleration Structures. The MBQ cross section is identical for the different types. In Fig. 1 a typical CLIC module (Type 1) is shown. The MBQ Type 1 is highlighted by the white circle (for clearness its supporting/stabilization structure is not present).

Manuscript received July 13, 2013; accepted October 8, 2013. Date of publication November 16, 2013; date of current version November 8, 2013. This work was supported in part by the European Commission under project EuCARD, Grant 227579.

The authors are with the CERN, 1211-Geneva, Switzerland (e-mail: michele. modena@cern.ch).

Color versions of one or more of the figures in this paper are available online at http://ieeexplore.ieee.org.

Digital Object Identifier 10.1109/TASC.2013.2285979

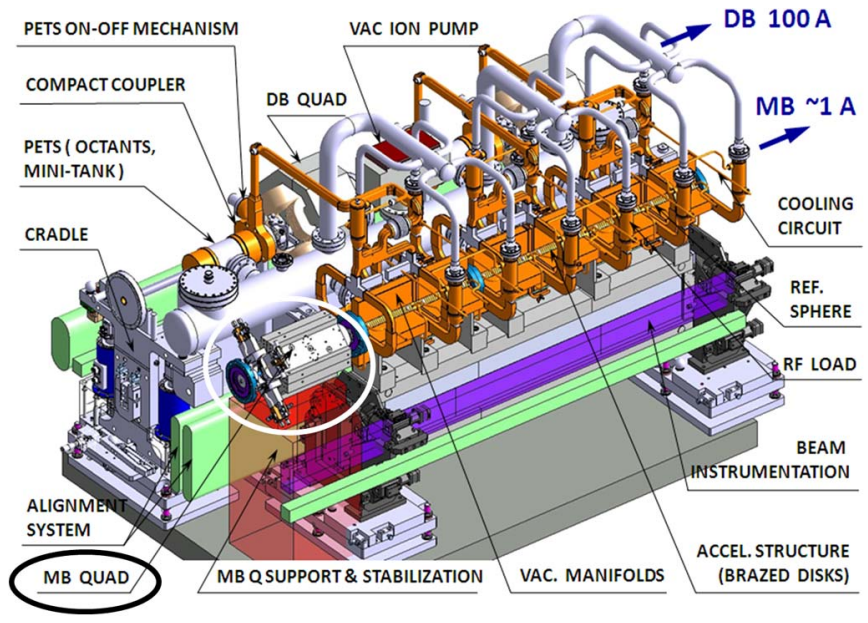

Fig. 1. Layout of a typical CLIC Module (here a Type 1 represented). Among the different components, the MBQ magnet circled in white are visible.

The MBQ design has to answer to several constraints and boundary conditions summarized here:

1) The quadrupoles have to provide a high gradient (200 T/m), and must be very compact in order to be integrated in the crowded layout of the CLIC Modules.

2) Since each magnet will be actively stabilized to the nanometer level [3], the mass of the magnet must be minimized and its stiffness maximized.

3) In order to provide the required magnetic field quality, the machining of the pole profile and the assembly of the quadrants have to keep very tight mechanical tolerances (target range is $5-10 \mu \mathrm{m}$ ).

4) Due to the large number of magnets to be procured (4020 units) any simplification in the design and assembly method would be extremely important.

\section{MBQ PRototypes Procurement Phases}

CERN started in 2009 a R\&D program on MBQ magnets. This phase of design and optimization has resulted in a set of design parameters for the four types of MBQ (see Table I for a subset of the main design parameters) and the procurement of prototypes (Type 1 and Type 4, the shortest and the longest of the MBQ family). Two of these prototypes are expected to be installed in the CLIC test facilities.

The first generation of MBQ Type 1 and Type 4 prototypes were procured in 2010-2011. The performances and measurements were good in terms of operational aspects and achieved magnetic gradient but not in terms of magnetic field quality (this was due to the machining of the iron quadrants that resulted in being out of specification). So, in 2011 a 
TABLE I

MAIN PARAMETERS OF MBQ FAMILY

\begin{tabular}{lllll}
\hline \hline Parameter & Value & & & \\
\hline Family & Type1 & Type2 & Type3 & Type4 \\
Quantity & 308 & 1276 & 964 & 1472 \\
Nominal field gradient [T/m] & 200 & 200 & 200 & 200 \\
$\begin{array}{l}\text { Nominal integrated field } \\
\text { gradient [T] }\end{array}$ & 70 & 170 & 270 & 370 \\
$\begin{array}{l}\text { Magnetic length [mm] } \\
\begin{array}{l}\text { Maximum overall length } \\
\text { [mm] }\end{array}\end{array}$ & 350 & 850 & 1350 & 1850 \\
$\begin{array}{l}\text { Magnet bore diameter [mm] } \\
\text { Good Field Region (GFR) }\end{array}$ & 10 & 10 & 10 & 10 \\
radius [mm] & 4 & 4 & 4 & 4 \\
$\begin{array}{l}\text { Integrated field gradient } \\
\text { error inside GFR }\end{array}$ & $<0.1 \%$ & & & \\
\hline \hline
\end{tabular}

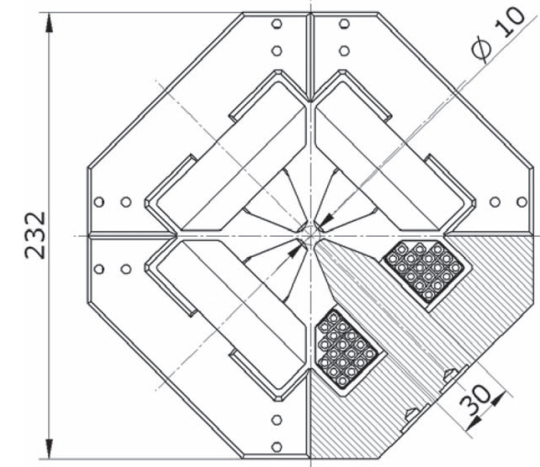

Fig. 2. MBQ second generation cross section.

second call was launched for tender with another selection of manufacturers. Achieving a good $\mu$-metric global precision in machining complex surfaces in iron blocks of $332 \mathrm{~mm}$ length (Type 1) is not easy and for the Type 4, where the length of the quadrant is $1827 \mathrm{~mm}$, it is even more challenging.

It was finally possible to procure two sets of Type 1 iron quadrants from two European companies, DMP Desarrollos Mecanicos de Precision (Gipuzkoa, Spain) and OSTROJ a.s. (Opava-Předměstí, Czech Republic), with conformity to tender mechanical tolerances. The electromagnetic coils were procured from the company TESLA Eng. Ltd (Storrington, United Kingdom).

\section{Manufacturing And Assembling of the MBQ}

Fig. 2 shows the quadrupole cross section.

The pole profile for this second generation prototypes was optimized with a set of circular sectors [2] that seems to provide a more uniform field quality for a small variation of the nominal current (gradient fine tuning).

The quadrants' critical surfaces, such as the pole profiles and the mating surfaces, were machined by a grinding technique. Stress relieving was performed at different steps during the manufacturing.

Other MBQ construction parameters for the Type 1 and Type 4 are shown in Table II. A second generation Type 4 prototype is still under procurement.

The magnet assembled with DMP quadrants is shown in Fig. 3.

The state-of-the-art technique for assembly of quadrants is based on the use of dowel pins (normally perpendicular to
TABLE II

MBQ Type1 AND Four Main CONSTRUCtion PARAMETERS

\begin{tabular}{lccc}
\hline \hline \multicolumn{1}{c}{ Parameter } & Type 1 & Type 4 & Unit \\
\hline Yoke length & 332 & 1827 & $\mathrm{~mm}$ \\
Nominal current & 140 & 128 & $\mathrm{~A}$ \\
Current density & 6.8 & 6.3 & $\mathrm{~A} / \mathrm{mm}^{2}$ \\
Windings per pole & 17 & 17 & \\
Conductor dimensions & $5.6 \times 5.6$ with hole Ø 2.5 & $\mathrm{~mm}$ \\
Total resistance & 48.2 & 220.3 & $\mathrm{~m} \Omega$ \\
Power & 0.95 & 3.61 & $\mathrm{~kW}$ \\
Cooling circuits/magnet & 1 & 4 & \\
Pressure drop & 5.9 & 6.3 & $\mathrm{bar}$ \\
Temperature rise & 15 & 15 & $\mathrm{~K}$ \\
\hline \hline
\end{tabular}

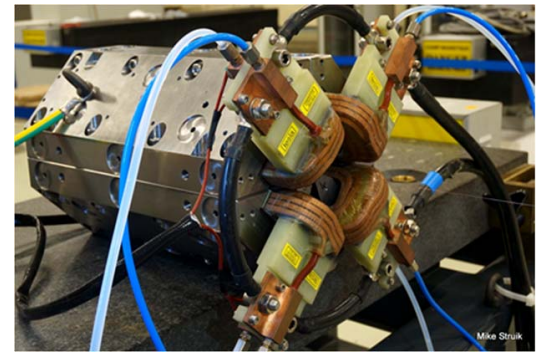

Fig. 3. Second generation MBQ Type 1 assembled.

the quadrants mating surfaces) and of other components like keys or stainless steel rings precisely fixed on the poles at the magnets extremities. Since one main scope of this R\&D and procurements is to investigate possible simplification in the machining and assembly (saving of cost and time for the expected large series production), it was decided to perform a first test assembling the quadrants only utilizing calibrated pins placed in the magnet bore and used as assembling tooling. Several sets of pins with small diameter differences up to 10 microns were used. To be mentioned is that at this conference we also report about a dedicated study to test the performances of different methods for precise magnet quadrants assembly [4].

Each quadrant, machined in solid steel AISI 1010 (AFNOR $\mathrm{XC10}$ ), was carefully inspected and measured at reception by the CERN Metrology laboratory.

Especially for the DMP quadrants excellent results were obtained in terms of the achieved tolerance. A global surface profile tolerance (according to ASME Y14.5-2009) of $\pm 7 \mu \mathrm{m}$ was obtained on the critical surfaces (pole profiles and quadrant mating surfaces). The graphs in Fig. 4 show some typical pole surface profile shapes measured on the DMP quadrants. The tolerance band lines are amplified and placed at $\pm 7 \mu \mathrm{m}$ with respect to the ideal pole profile.

The quadrants from OSTROJ were measured to be inside a tolerance limit of $\pm 30 \mu \mathrm{m}$.

After assembly, the two magnet cores were measured by the CERN Metrology lab in order to determine the precision of the quadrants assembly. The results obtained are:

- OSTROJ quadrants: the assembly of the four poles surfaces profile (according to the previously mentioned ASME norm) is inside a tolerance of $\pm 127 \mu \mathrm{m}$.

- DMP quadrants: inside a tolerance of $\pm 177 \mu \mathrm{m}$. 


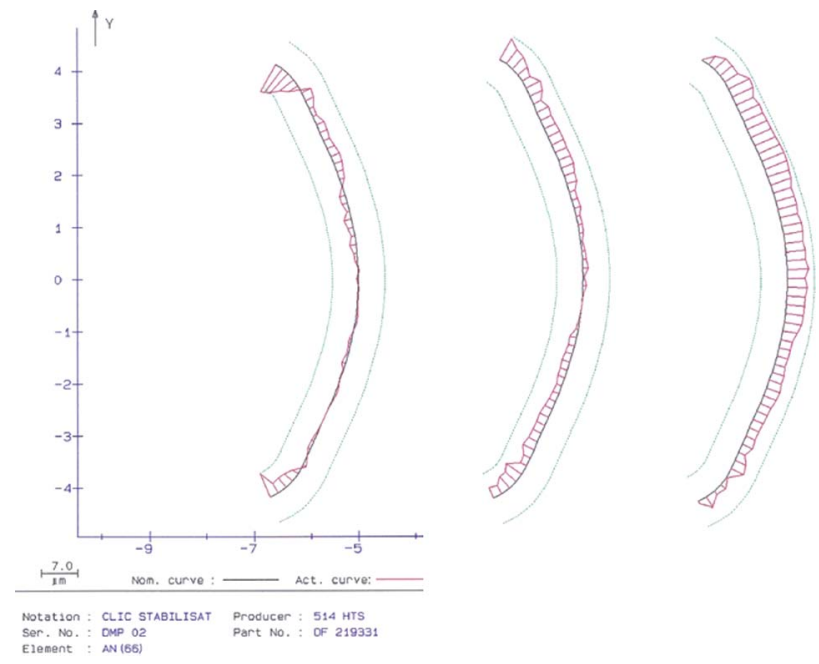

Fig. 4. Three typical metrology measurements of the DMP quadrants pole profiles: Tolerance bands positioned at $\pm 7 \mu \mathrm{m}$.

\section{Magnetic Measurements of the MBQ}

A main interest was to check the influence of the quadrants machining and achieved assembly precision in the two procurements on the magnetic field quality.

For this reason, the two prototypes were recently measured at CERN by stretched and oscillating wire methods [5]. In fact, due to the very small magnet aperture ( $5 \mathrm{~mm}$ radius), these are the only methods available today at CERN to measure gradient, magnetic axis, and field multipoles expansion. A rotating coil system with diameter of $7.4 \mathrm{~mm}$ is under development at CERN but not yet operational.

The analysis of the measurement results is not straightforward, and the following aspects are obvious:

- Despite the fact that the DMP quadrants are better machined individually, the first (and unique for the moment) quadrants assembly test has yielded a better overall field quality for the magnet assembled with the OSTRJ quadrants.

- The longitudinal grinding reproducibility is very good for both produced sets of quadrants and it is inside a limit of $\pm 2 \mu \mathrm{m})$. This good quality aspect must be carefully evaluated since it can have also a negative impact on the global magnet field quality. In fact, a good longitudinal reproducibility means also that eventual pole surface shape errors (for example the second and third profiles of Fig. 4) will be longitudinally less randomized. This could cause the appearance of some systematic errors in the integrated field quality as measured along the entire magnet length (as done by the stretched wire or later seen by the particle beam).

In Fig. 5 the shift of the magnetic center as a function of the powering current is shown. The magnet was precycled five times and then the shift of the magnetic center was measured all along the following cycle: starting from a current of $60 \mathrm{~A}$, then up to $I_{\max }$, then down to $-I_{\max }$ and repeating twice this current cycle. It is notable how the magnetic center is shifting in a reproducible way inside a limit of $\pm 12 \mu \mathrm{m}$ (the zero of the magnetic center is arbitrarily set at the nominal current value of $140 \mathrm{~A})$.

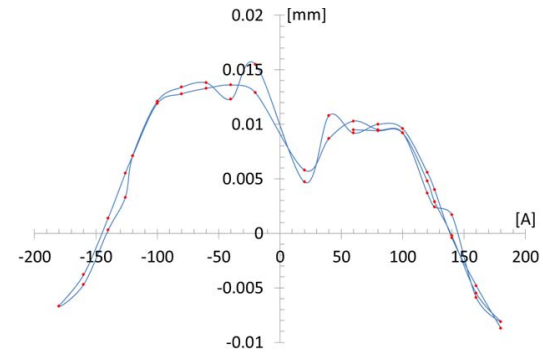

Fig. 5. Magnetic center shift in function of powering current.

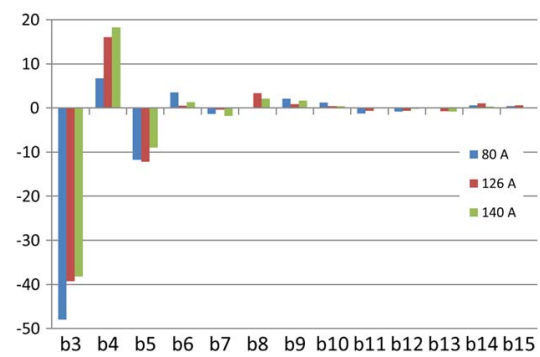

Fig. 6. MBQ (DMP version) normal magnetic multipoles.

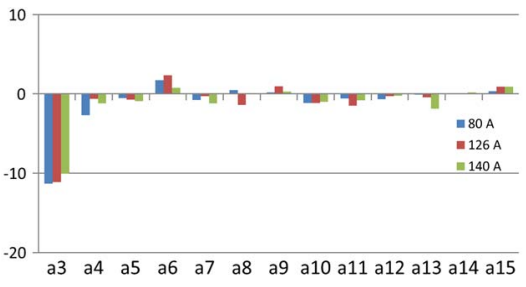

Fig. 7. MBQ (DMP version) skew magnetic multipoles.

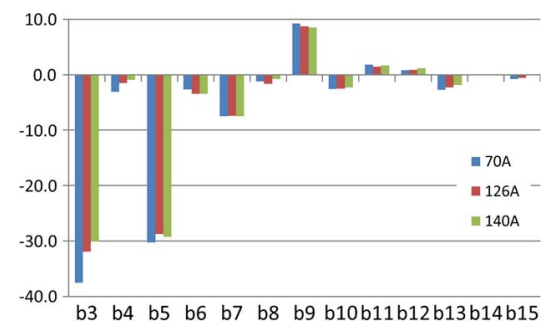

Fig. 8. Normal magnetic multipoles for MBQ (Ostroj version).

In Figs. 6 and 7 are reported the multipole contents (normal and skew) for the magnet assembled with the DMP quadrants.

The first "permitted" normal multipoles are $b_{6}$ and $b_{10}$ (the multipole unit defined as a factor $10^{-4}$ respect to the main $\left(b_{2}\right)$ component). For this specific magnet design, the expected value for these two components (in the case of a "perfect" magnet) is about 1 unit; this was assessed during the design phase by the Finite Element Analysis OPERA code.

Magnetic measurements were done at a radius of $4 \mathrm{~mm}$ (the radius specified for the GFR, see Table I) which is $80 \%$ of the available aperture. The oscillating wire system is still experimental but its performance and cross-checks done with other systems (rotating coils) indicate an expected precision of \pm 5 units.

Figs. 8 and 9 show the results of similar measurements done for the magnet assembled with the OSTROJ quadrants.

The following remarks can be made:

I. As well-known [6], [7], all the measured "forbidden" multipoles are due to quadrants machining and assembly imperfections. In both assemblies (DMP and OSTROJ 


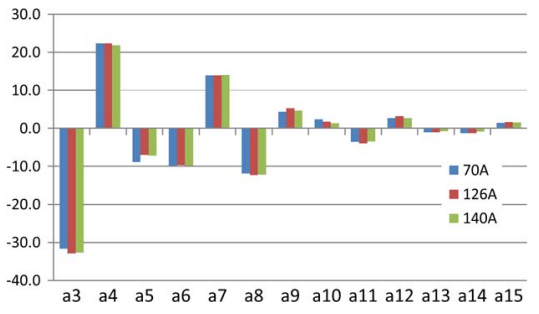

Fig. 9. Skew magnetic multipoles for MBQ (Ostroj version).

quadrants) forbidden multipoles are present. To a certain extent this fact is unavoidable because of the link between the physical limits of the machining precision and the precision to which the core assembly can be made. Even in the case of the very good $\pm 7 \mu \mathrm{m}$ surface profile obtained with the DMP quadrants, the lack of symmetry amongst the assembled 4 quadrants will lead to forbidden multipoles.

Analyzing the different multipoles components content we found a good agreement with the metrology results obtained for the two assembled magnets.

For example: in the DMP assembly two opposite poles were measured as displaced along their radii (one inward and the outer outward) of about 140 and $150 \mu \mathrm{m}$. Using formulae in [6] and [7] such displacements will lead to strong sextupole and some decapole components. In a similar way a relative larger rotation along its longitudinal axis was measured especially for 1 pole. This should explain the presence of the decapole $b_{5}$ and octupole content. In a similar way it is possible to trace back to the origin of the other measured main forbidden multipole components measured.

II. Despite being less precisely assembled, the multipoles content for the DMP is globally better with respect to the OSTRJ (especially for the skew components). This can be explained by the better individual machining of this quadrants set.

III. The $\mathrm{b}_{6}$ and $\mathrm{b}_{10}$ component contents are limited. This proves the validity of the pole shape design choice and of the overall machining quality achieved on the poles surfaces.

IV. It is also interesting to remark the coherence between these assembly results and the conclusion of the test program on dummy pieces [4].

Our future plans are on two fronts:

a. Continue to investigate alternative and cheaper methods for assembling compact quadrupole quadrants. Since an additional good reference is the lateral distance between adjacent poles we would like to test the assembly of the quadrants with the help of some special tooling that will include a precise cylindrical part (pin) on which the poles inner surfaces will be aligned, and a more complex radial part that will match the four lateral surfaces of the poles. The details of these tooling are now under definition and design.

b. To machine on the DMP quadrants a set of longitudinal slots to be used as of alignment keys (as done in some standard mechanical precision assemblies). This solution would increase the cost of the machining but if longitudinal slots would be sufficient and the use of perpendicular dowel pins could be avoided, it would be anyway advantageous compared to more classical solutions.
In the case of a successful implementation of these solutions we expect to measure on the DMP prototype evidence of decreasing $a_{3}$ and $b_{3}$ components plus the reduction of other multipoles. This should also help to better discern between core assembly errors and effects due to achieving the limits on pole machining tolerances (cfr. Fig. 4). Even if the differences from perfect profile are very small, they will have a noticeable impact on field quality due to the relatively small magnet bore aperture.

\section{CONCLUSION}

The design and procurement of CLIC MBQ prototypes have recently advanced with the final assembly and measurement of two new Type 1 prototypes, assembled with two different solid iron quadrants productions.

The iron quadrants were machined close to the best achievable precision; especially the quadrants of one company (DMPSpain) are reaching an excellent global surface profile tolerance of $\pm 7 \mu \mathrm{m}$.

The mechanical measurements performed by the CERN metrology laboratory on the individual and the assembled quadrants, show good agreement with magnetic measurements multipole results obtained with the stretched and oscillating wire methods.

One of the most interesting aspects of this $R \& D$ is to try to identify alternative and cheaper methods for the very precise assembly of small quadrupole quadrants. The metrology and magnetic measurements have shown how, despite the very good individual machining tolerance achieved, the core assembly method tested is not precise enough and that the assembly procedure needs further investigation and improvements.

Future plans consist of:

a. Design and test special assembly tooling to better center the pole reference surfaces.

b. To modify one quadrant set to test assembling with simplified longitudinal keys.

A main goal remains to soon provide to the CLIC project management a good estimate of the achievable field quality versus the price for these very compact magnets (inner bore radius: $5 \mathrm{~mm}$ ) that have to be procured in more than 4000 units.

\section{REFERENCES}

[1] CLIC Conceptual Design Report, CERN, Meyrin, Switzerland. [Online]. Available: http://clic-study.org/accelerator/CLIC-ConceptDesignRep.php

[2] A. Vorozhtsov and M. Modena, "Design and manufacture of a main beam quadrupole model for CLIC," IEEE Trans. Appl. Supercond., vol. 22, no. 3, p. 4002404, Jun. 2012

[3] S. Janssens, K. Artoos, C. Collette, M. Esposito, P. Fernandez-Carmona, M. Guinchard, and R. Leuxe, "Stabilization and nano-positioning of the CLIC main-beam quadrupoles," presented at the International Conference on Noise and Vibration engineering (ISMA2012)-International Conference on Uncertainty in Structural Dynamics (USD2012), Leuven, Belgium, 2012.

[4] M. Modena, R. Leuxe, and M. Struik, "Results on ultra-precise magnet yoke sectors assembly tests," presented at the 23rd Int. Conf. Magnet Technol., Boston, MA, USA, Jul. 2003, 163991.

[5] P. Arpaia, C. Petrone, M. Buzio, S. Russenschuck, and S. L. Walckiers, "Multipole correction of stretched-wire measurements of field-gradients in quadrupole accelerator magnets," J. Instrum., vol. 8, 2013, Ref: 2013 JINST 8 P08010.

[6] K. Halbach, "First order perturbation effects in iron-dominated twodimensional symmetrical multipoles," Nucl. Instrum. Methods, vol. 74, no. 1 , pp. 147-164, Oct. 1969.

[7] Jack and T. Tanabe, Iron Dominated Electromagnets: Design Fabrication, Assembly and Measurements. Singapore: World Scientific, 2005, ch. 4. 